\title{
Sudden Cardiac Death in Young Athletes
}

Dilip R Patel*, MD

Author's Affiliation: Michigan State University College of Human Medicine, Sports Medicine Program, Kalamazoo Center for Medical Studies, USA

Address: Kalamazoo Center for Medical Studies, 1000 Oakland Drive Kalamazoo Michigan 49008, USA

E-mail: patel@kcms.msu.edu

$\mathrm{T}$ article by Halabchi et al. provides a comprehensive, thoughtful and balanced analysis of the literature on sudden cardiac death (SCD) in young athletes ${ }^{[1]}$. The article is unique in providing a special perspective for Asia as contrasted with those in Europe and the United States. This aspect is extremely important to recognize for the management of young athletes in different parts of the world.

In the United States, the history and physical examination (H\&P) has been the standard of cardiovascular screening for competitive athletes, although several studies have questioned the efficacy of the screening H\&P alone in identifying athletes at risk for cardiovascular adverse events ${ }^{[2-6]}$. The H\&P has come under scrutiny for having low sensitivity for identifying athletes with cardiovascular risk factors. Several studies have reported data substantiating the higher efficacy of electrocardiogram (ECG) in identifying athletes with hypertrophic cardiomyopathy (HCM), channelopathies, and other clinically silent cardiovascular diseases that increase the risk for SCD in young athletes ${ }^{[2,6-12]}$.

The Italian experience using the ECG as part of screening athletes is cited most frequently as the basis for including ECG as a screening tool ${ }^{[4]}$. One argument against that study is that it is an observational study and not a prospective study with a control population ${ }^{[3]}$. At present there is no worldwide consensus on the added value of including ECG as a screening tool for all young athletes. In the United States the American Heart Association report provides the general framework for cardiovascular screening ${ }^{[3,5]}$. Large prospective studies are needed to evaluate the value of screening ECGs in the US population.

2-D echocardiogram (ECHO) is most useful for the diagnosis of structural heart disease ${ }^{[13]}$. Adding an ECHO to a cardiovascular screening program increases the likelihood of identifying structural abnormalities of the heart. The added cost of screening and the scarcity of resources, equipment, and personnel are significant barriers to recommend routine use of ECHO for screening. An ECHO can be considered a good confirmatory test after abnormal findings on H\&P or ECG.

There have been significant advances in the identification of genetic causes for cardiovascular diseases, leading to the question of present or future methods involving identification of genetic risk factors for cardiovascular disease using genetic testing as a screening tool ${ }^{[14,15]}$. Exercise stress testing is of limited value as a screening tool but has a role in risk stratification $^{[13]}$. Exercise stress testing has been recommended in athletes older than 35. This is also part of the extended Italian recommendation for athletes older than $35^{[4,5]}$. Maximal exercise testing is an integral component to unmask arrhythmia in arrythmogenic ventricular cardiomyopathy (ARVC), familial catecholaminergic polymorphic ventricular tachycardia, and long QT syndrome. Frequent ventricular extrasystoles during exercise indicate underlying cardiac disease ${ }^{[13]}$. Several other tests are available for identification of certain specific conditions ${ }^{[13,15]}$. These include Holter 
monitoring (quantification of extrasystoles and identification of tachy-and bradyarrythmias), cardiac magnetic resonance imaging (MRI) scan (ARVC, HCM, and myocarditis), tissue Doppler (ARVC), sodium channel blocker challenge (Brugada syndrome), and adenosine challenge for pre-excitation (familial risk of WPW syndrome and supraventricular tachycardia). These tests are not appropriate for mass screening due to low yield and also due to the need for expertise to successfully obtain and interpret the results. They become important after screening has raised concerns for specific problems.

Also as shown by Hibachi et al ${ }^{[1]}$, there are a number of regional considerations in terms of the size of the young athlete populations, epidemiology of sudden cardiac death, access to technology, and economic feasibility. A world wide collaborative effort is needed to further delineate various disparate considerations pertaining to SCD in young athletes so that a more uniform, cohesive and regionally appropriate screening and prevent ion strategy can evolve.
Identification of young athletes at risk for an adverse cardiovascular event during sport participation is a challenging task ${ }^{[2]}$. Even in the best of circumstances, outcome of sudden cardiac arrest (SCA) on the field remains poor; therefore, prevention is critical. Cardiovascular screening of young athletes is based on ascertaining specific history and a thorough physical examination. Although inclusion of an ECG in screening asymptomatic athletes has been shown to increase the likelihood of identifying athletes at risk, considerable logistical and economic challenges remain, including using ECG as a screening tool. Even the most ideal screening strategy may not identify all athletes at risk for SCA on the field, and unfortunately SCD may be the first clinical manifestation of some cardiac conditions ${ }^{[2]}$.

\section{Conflict of interests: None}

Key Words: Sudden Cardiac Death; Sudden Cardiac Arrest; Athletes

\section{REFERENCES}

1. Halabchi F, Seif-Barghi T, Mazaheri R. Sudden cardiac death in young athletes; a literature review and special considerations in Asia. Asian J Sports Med 2011;2:1-16.

2. Siddiqui SA, Patel DR. Prevention of sudden cardiac death in young athletes: impact and limitations of preparticipation screening. Int Public Health J 2009;1:379-88.

3. Maron BJ, Thompson PD, Ackerman MJ, et al. Recommendations and considerations related to preparticipation screening for cardiovascular abnormalities in competitive athletes: 2007 update. Circulation 2007;115:1643-55.

4. Corrado D, Pelliccia A, Bjornstad HH, et al. Cardiovascular pre-participation screening of young competitive athletes for prevention of sudden death: proposal for a common European protocol. Consensus Statement of the Study Group of Sport Cardiology of the Working Group of Cardiac Rehabilitation and Exercise Physiology and the Working Group of Myocardial and Pericardial Diseases of the European Society of Cardiology. Eur Heart J 2005;26:516-24.

5. American College of Cardiology 36th Bethesda Conference: eligibility recommendations for competitive athletes with cardiovascular abnormalities. J Am Coll Cardiol 2005;45:1317-75.

6. Corrado D, Basso C, Schiavon M, et al. Pre-participation screening of young competitive athletes for prevention of sudden cardiac death. J Am Coll Cardiol 2008;52:1981-9.

7. Corrado D, Basso C, Pavei A, et al. Trends in sudden cardiovascular death in young competitive athletes after implementation of a preparticipation screening program. JAMA 2006;296:1593-601.

8. Bille K, Figueiras D, Schamasch P, et al. Position paper: sudden cardiac death in athletes: the Lausanne recommendations. Eur J Cardiovasc Prev Rehabil 2006;13:859-75.

9. Corrado D, McKenna WJ. Appropriate interpretation of the athlete's electrocardiogram saves lives as well as money. Eur Heart J 2007;28:1920-2.

10. Myerburg RJ, Vetter VL. Electrocardiograms should be included in preparticipation screening of athletes. Circulation 
2007;116:2616-26.

11. Pelliccia A, Culasso F, Di Paolo FM, et al. Prevalence of abnormal electrocardiograms in a large, unselected population undergoing pre-participation cardiovascular screening. Eur Heart J 2007;28:2006-10.

12. Corrado D, Pelliccia A, Heidbuchel H, et al. Recommendations for interpretation of 12-lead electrocardiogram in the athlete. Eur Heart J 2010;31:243-59.

13. Attari M, Dhala A. Role of invasive and noninvasive testing in risk stratification of sudden cardiac death in children and young adults: an electrophysiologic perspective. Pediatr Clin North Am 2004;51:1355-78.

14. Tan H, Hofman N, van Langen I, et al. Sudden unexplained death: heritability and diagnostic yield of cardiological and genetic examination in surviving relatives. Circulation 2005;112:207-13.

15. Prakken NH, Velthuis BK, Cramer MJ, et al. Advances in cardiac imaging: the role of magnetic imaging and computed tomography in identifying athletes at risk. Br J Sports Med 2009;43:677-84. 